\title{
Role of fine needle aspiration cytology in assessment of cervical lymphadenopathy
}

\author{
Kochhar A.K ${ }^{1}$, Puri P.L ${ }^{2}$, Kochhar $S^{3}$ \\ ${ }^{1}$ Dr. Ajay Kumar Kochhar, Associate Professor, Pathology Department, Maharaja Agrasen Medical College, Agroha, \\ Hisar, Haryana, ${ }^{2}$ Dr. Prem Lata Puri, Associate Professor, Physiology, Department, Maharaja Agrasen Medical College, \\ Agroha, Hisar, Haryana, ${ }^{3}$ Dr. Sonia Kochhar. Professor, Physiology Department, MMIMSR, Mullana, Ambala, Haryana, \\ India.
}

Address for correspondence: Dr. Ajay Kumar Kochhar, Email: ajaykochhar10@yahoo.com

\begin{abstract}
Background: Lymphadenopathy is one of the commonest clinical presentations of patients attending the outdoor department. The causes vary from simple treatable infections to malignancies and hence need to be speedily investigated. Fine needle aspiration cytology (FNAC) is simple, quick, inexpensive and minimally invasive technique that can be used as an outpatient procedure to diagnose them. The Aim and objective of this study is to assess the various causes of cervical lymphadenopathy through FNAC, and to see the distribution of lesions with respect to age and gender. Method: The present randomized study was undertaken to study non neoplastic and neoplastic lesions of enlarged cervical lymph nodes by FNAC in 135 patients presented with cervical lymphadenopathy in Shree Krishna Hospital, Karamsad over a period of March 2006 to June 2007. Result: Tuberculous lymphadenitis, reactive lymphadenopathy, suppurative lymphadenitis, lymphoma, leukemic infiltration and metastatic lymphadenopathy were seen in 38.52\%, 24.45.\%, 3.70\%, $2.22 \%, 0.74 \%$ and $30.37 \%$ respectively. Tuberculous lymphadenitis was seen most often in third decade and reactive hyperplasia in first two decades of life. Metastatic lymphadenopathy was seen predominantly above forty years of age. Metastatic lesions of lymph nodes were seen to be more common in males. Squamous cell carcinoma is the most common metastatic lesion. Conclusion: The present study highlights the usefulness of FNAC in early diagnosis in cases presenting with cervical lymphadenopathy. It can differentiate a neoplastic from a non-neoplastic process and therefore influence the patient management and prevent them from being subjected to unnecessary surgery.
\end{abstract}

Keywords: Cervical lymphadenopathy, Fine needle aspiration cytology, Tuberculous lymphadenitis, Lymphoma.

\section{Introduction}

Cervical Lymphadenopathy is one of the commonest clinical presentation of patients attending the outdoor department. Fine needle aspiration cytology is a reliable, simple, safe, rapid and inexpensive method of establishing the diagnosis of cervical lymphadenopathy [1]. It also gives a great relief to the anxiety of the patients and relatives as it is a safe, almost painless requiring no anaesthesia and the report is available within a short time avoiding complications [2]. The role of FNAC in the investigation of lymphadenopathy has previously been established by a number of studies [3-8]. Due to the simplicity of the technique and speedy results, FNAC needs to be promoted. The most

\footnotetext{
Manuscript received $27^{\text {th }}$ April 2016

Reviewed: $6^{\text {th }}$ May 2016

Author Corrected: $16^{\text {th }}$ May 2016

Accepted for Publication 29 May 2016
}

common cause of peripheral lymphadenopathy in our study is an inflammatory reaction to a microbial challenge, followed by lymphomas and malignant metastatic deposits. The etiological factors for these lesions make a large list. Broadly speaking, they tend to be considered as reactive, tuberculous, primary lymphoid malignancy like lymphomas and malignant metastases. Overall, infective conditions (reactive and tuberculous) are responsible for the majority of lesions. Lymph nodes clinically suspected of lymphomas or malignant metastasis is one of the most common indications for FNAC in the elderly. The biopsy of the cervical lymph node is always the gold standard. However, it is more time consuming than FNAC, requiring anaesthesia, strict asepsis, and theatre time and often leaves a scar. In contrast, FNAC of the 
cervical lymph node is relatively simpler and offers quick reliable results.

Aims and Objectives: To assess the various causes of cervical lymphadenopathy through FNAC, and to see the distribution of lesions with respect to age and gender.

\section{Material and Method}

A total of 135 patients presenting with superficial palpable cervical lymph nodes, who were referred to cytology section of pathology department from the OPD/IPD of Shree Krishna hospital, Karamsad, were studied over a period of sixteen months. In each instance, a brief history and physical examination along with evaluation of relevant investigation, if available, was carried out. FNAC procedure performed by pathologist using 22-24 G needle attached to $10-20 \mathrm{ml}$ of syringe. Aspirated material was smeared onto slides in each case.

Slides were immediately put into the fixative solution and air dried. Alcohol fixed smears were stained by Hematoxylin and eosin and Papanicolaou method. The air dried smears were stained with May-GrunwaldGiemsa (MGG) stain. Special stains like Ziehl Neelson (ZN) stain for acid fast bacilli and Periodic Acid Schiff (PAS) stain was used whenever required.

\section{Results}

This study analyzed 135 cases of cervical lymphadenopathy. The age of patients ranged from 8 months to 88 years in which $54.07 \%$ were males and $45.93 \%$ were females [Table 1]. Cases with palpable lymph nodes, Tuberculous lymphadenitis, reactive lymphadenopathy, suppurative lymphadenitis, lymphoma, leukemic infiltration and metastatic lymphadenopathy were seen in $38.52 \%, 24.45 \%, 3.70 \%, 2.22 \%, 0.74 \%$ and $30.37 \%$, respectively (Table-II). Tuberculous lymphadenitis was the most common cause of lymphadenopathy followed by metastatic lesions. Tuberculous lymphadenitis was seen in 52 Patients. Tuberculous lymphadenitis was seen often in second and third decades of life. (Table-III) Smear from tuberculous lymphadenitis show on epithelioid granulomas, caseous necrosis with or without AFB., sometimes show extensive necrosis with AFB positive (on ZN stain or culture). Reactive lymphadenitis was seen in 33 patients. Their aspirate showed the various stages of maturing lymphocytes, as seen in a reactive lymphoid follicle. In this group some patients showed features of viral infection as prominence of immunoblasts and plasma cells. The background of lymphocytes was however polymorphous. The peripheral blood smear in these cases showed antigenically stimulated lymphocytes. In a follow up of these cases over a period of 6 months, the clinical and peripheral blood findings totally regressed. Reactive hyperplasia was seen in first two decades of life.

There were three patients of lymphoma. The aspirate in one case was highly cellular and showed an almost monotonous population of round cells with scanty cytoplasm which were larger than lymphocytes. In two cases, the background population of lymphoid cells was polymorphous, but they showed few large cells, reminiscent of Reed-Sternberg cells. Presence of epithelial cells in lymph node is pathognomic of metastatic carcinoma, as normal lymph node does not have any epithelial cells.

The cytological appearance of Squamous cell carcinoma depends on degree of differentiation and the associated degenerative changes by the tumour. Keratinizing squamous cell carcinoma are readily identified when cells with abundant, sharply demarcated, dense eosinophilic cytoplasm and pyknotic nuclei are present in the smears. Anucleated squames may also appear, as may spindle shaped or tadpole shaped cells, arranged in cell- within-cell pattern. Occasionally, keratin forming cells with small pyknotic nuclei are present without the typical features of cancer cells.

Table-1: Age and Gender Distribution of 135 cases of Cervical Lymphadenopathy.

\begin{tabular}{|l|l|l|l|}
\hline Age group (years) & Males & Females & Total \\
\hline $0-20$ & 14 & 15 & 29 \\
\hline $21-40$ & 12 & 28 & 40 \\
\hline $41-60$ & 26 & 14 & 40 \\
\hline$>61$ & 21 & 05 & 26 \\
\hline
\end{tabular}


Table-2: Cytological diagnosis of Lymphadenopathy.

\begin{tabular}{|l|l|l|}
\hline Cytological Diagnosis & No. of Cases & Percentage (\%) \\
\hline Tuberculous lymphadenitis & 52 & 38.52 \\
\hline Reactive lymphadenitis & 33 & 24.45 \\
\hline Suppurative lymphadenitis & 05 & 3.70 \\
\hline Lymphoma & 03 & 2.22 \\
\hline Leukemic Infiltration & 01 & 0.74 \\
\hline Metastatic & 49 & 30.37 \\
\hline
\end{tabular}

Table-3: Distribution of cases.

\begin{tabular}{|l|l|l|l|l|l|l|l|l|}
\hline \multirow{2}{*}{} & \multirow{2}{*}{ Cytologic Diagnosis } & \multicolumn{3}{|l|}{$\mid$} & & \\
\cline { 3 - 10 } & & & & & & & & \\
\hline 1 & Tuberculosis Lymphadenitis & 03 & 11 & 16 & 08 & 06 & 05 & 03 \\
\hline 2 & Reactive hyperplasia & 06 & 09 & 08 & 03 & 04 & - & 03 \\
\hline 3 & Suppurative Lymphadenitis & 01 & - & 01 & 01 & 01 & - & 01 \\
\hline 4 & Lymphoma & 01 & - & - & 01 & - & - & 01 \\
\hline
\end{tabular}

Table 4: Comparison of the incidence of lymphadenopathy due to different aetiological factors.

\begin{tabular}{|l|l|l|l|l|}
\hline $\begin{array}{l}\text { Aetiological } \\
\text { Group }\end{array}$ & Patra et al [5] & Bhaskaran et al [4] & Khajuria et al [6] & Present Study \\
\hline $\begin{array}{l}\text { Tuberculous } \\
\text { Lymphadenitis }\end{array}$ & $37.8 \%$ & $67.57 \%$ & $52.3 \%$ & $38.52 \%$ \\
\hline Reactive Lymphadenopathy & $33.0 \%$ & $20.86 \%$ & $37.2 \%$ & $24.45 \%$ \\
\hline Suppurative Lymphadenitis & $5.8 \%$ & $1.5 \%$ & $1.0 \%$ & $3.70 \%$ \\
\hline Lymphoma & $6.7 \%$ & $2.97 \%$ & $2.0 \%$ & $2.22 \%$ \\
\hline Leukemic Infiltration & $0.9 \%$ & - & - & $0.74 \%$ \\
\hline Metastatic Carcinoma & 14.5 & 5.6 & 3.8 & $30.37 \%$ \\
\hline
\end{tabular}

Metastatic lymphadenopathy was seen predominantly above forty years of age. Cervical lymph nodes were involved in all types of lymphadenopathy. Squamous cell carcinoma is the most common metastatic lesions of lymph node. Metastatic cervical lymphadenopathy was seen in forty one patients. Out of 41 cases, 38 cases were of metastatic squamous cell carcinoma, two cases showed evidence of metastatic adenocarcinoma and the remaining one case showed metastatic papillary carcinoma of thyroid. Metastatic lesions of lymph node are more common in males as compared to female. Cervical lymphnode is the most common site for metastasis of squamous cell carcinoma.

\section{Discussion}

Fine Needle aspiration (FNAC) is inexpensive, completely safe and quick method for diagnosis of lymphadenopathy and it reduces the need for surgical biopsy. We have presented our experience with 135 cases of lymphadenopathy over a period of Sixteen months. In the present study, diagnosis was based on definite cytomorphological findings with cliniccytological co-relation. Our primary aim was to help the clinician in arriving at an early diagnosis in cases presenting with lymphadenopathy. The pattern of lesions consisted of tuberculous lymphadenitis, reactive lymphadenopathy, suppurative lymphadenitis, lymphoma, leukemic infiltration and metastatic lymphadenopathy seen in our study is more or less is same as reported in other studies in India and other developing countries [2,5,6]. Maximum numbers of cases in our study are of tuberculous lymphadenitis. In India, tuberculous lymphadenitis is one of the most common type of lymphadenopathy encountered in clinical practice $[4,7,8,9]$. Whereas it is in sharp contrast to very low frequency of $1.6 \%$ in developed countries [10]. The highest incidence of tuberculous lymphadenitis was seen in third decade of life. Patra et al [5] had 37.8\% cases of tuberculosis while the present study has $38.52 . \%$ cases. This was quite close to our studies. Similar study was done by Khajuria et al [6] which show Tubercular lymphadenitis was $52.3 \%$ and 
Bhaskara et al [4] showing $67.57 \%$ (Table IV). All these authors studies including our study suggest that Tubercular lymphadenitis is the most common cause of lymphadenopathy. The discrepancy in results is due to wide variation in study age group and socio-economic condition of the patients. In present study $53.33 \%$ of cases are contributed by patients less than 40 years while study done by Khajuria [6] show $79.57 \%$, significant contribution, in this age group. Reactive hyperplasia was seen in first two decades of life. Metastatic lymphadenopathy was seen predominantly above the forty years of age. Metastatic malignancies are significantly more common in males. The superficial cervical lymphnodes are common sites of metastasis. Squamous cell carcinoma is the most common metastatic lesion. In diagnosis of metastatic malignancy, lymph node aspiration is as rewarding as surgical biopsy. Metastatic carcinoma was observed in $5.6 \%$ cases of Bhaskara et al [4], $14.5 \%$ of cases by Patra AK et al [5] and 3.8\% cases by Ruchi Khajuria [6] and which is in sharp contrast to our studies which shows $30.37 \%$ of metastatic carcinoma. This variation in results is due to same reason, that is, difference in age groups of patients, as most of metastatic lesions are common above 40 years of age. In Patra's study, majority of patients, $43.68 \%$ are in the first decade, while in contrast, majority of patients $(47.33 \%)$ in our study are above 40 years. Patra restricted his study up to 60 years of age as compared to our study which includes $17.78 \%$ of cases above 60 years of age. In study done by khajuria [6], patients above 40 years of age constitute $15.70 \%$ of cases. This shows significant difference and contribute to this variation. The other reason for higher metastasis is the regional variation, as in this region tobacco farming is more prevalent and hence tobacco chewing, which is one of the major predisposing factor for malignancy especially of aero digestive tract. The third reason for getting higher percentage of metastatic lesions in our study as compared to other references may be due to the fact that there is a cancer and radiotherapy centre in our institution; hence we are getting more referrals for malignancies.

The anatomical site of involved node along with age and sex may give some indication to the location of primary tumour. For example, Upper cervical lymph nodes commonly harbours the metastatic deposits from aerodigestive tract and mid cervical harbours metastasis from thyroid. The cytomorphological pattern seen in routinely stained smears often give clue to the site of primary tumour. Intranuclear cytoplasmic inclusions suggest metastasis from papillary carcinoma of thyroid. Typical microacinar formation suggests metastatic from occult follicular carcinoma of thyroid. Plasmacytoid, triangular or spindle shaped cells with intracytoplasmic granules, suggest metastasis from medullary carcinoma of thyroid. Small monmorphic tumor cells surrounding globules or cylinders suggest adenoid cystic carcinoma. Glandular cells moderately pleomorphic arranged in a gland - in - gland or in cribriform pattern suggest a prostatic carcinoma. Columnar cells with elongated nuclei arranged in pallisades, stringy mucus and necrosis suggests primary in large bowel, while mucin containing signet ring cells suggest the stomach as the most likely primary site among several other possibilities. In present study squamous cell carcinoma is the most common metastatic lesion of lymph node which is comparable with other studies $[4,11,12]$.

\section{Conclusion}

The present study highlights the usefulness of fine needle aspiration cytology that fulfilles the primary aim to help the clinician in arriving at early diagnosis in cases presenting with cervical lymphadenopathy. It is easy, may be performed in OPDs, and serves as a rapid modality for the diagnosis of one of the common curable causes of cervical lymphadenopathy as tuberculosis. A cytological follow up of the lesions is also easily possible by FNAC study. Tuberculosis stands out to be the most common cause of cervical lymphadenopathy in India. Patients with a heavy acidfast bacilli load in the aspirate should be screened for HIV infection. FNAC may be the only tool available for the diagnosis of metastatic malignant lesions in lymph nodes in disseminated cancer and malignancies with an occult primary. For the diagnosis of lymphomas, FNAC can strongly suggest a preliminary diagnosis, which can be followed by biopsy for histopathology and immunohistochemistry for confirmation and final classification. FNAC helps in early diagnosis and save the patients from high mortality and morbidity especially in malignant diseases.

Funding: Nil, Conflict of interest: None initiated. Permission from IRB: Yes

\section{References}

1. Harsh Kumar, Shirish S. Chandanwale, Charusheela R. Gore Role of fine needle aspiration cytology in assessment of cervical lymphadenopathy Medical Journal of Dr. D.Y. Patil University 2013; 6 :(4) : 400404. 
2. Russel J. Orell, S. Skinner, J. Sehsadri R. Fine needle aspiration cytology in the management of lymphoma, Aust NZ J. Med. 1983 Aug; 13(4): 365 -68.

3. Arora B, Beena KR. Utility of FNAC in Lymphadenopathies. J. of Cytology. 1999; 16(2):61-66.

4. Bhaskaran CS, Kumar HG, Sreenivas M. Kamleshwari, R. Rao G, Aruna CA. Fine needle aspiration cytology. Review of 1731 cases. Indian J. Pathol Microbiol. 1990 Oct; 33(4): 387- 97.

5. Patra AK, Nanda BK, Mohapatra BVK, Pnada AK. Diagnosis of lymphadenopathy by fine needle aspiration cytology. Indian J. Pathol. Microbiol. 1983 Oct; 26(4):273-278.

6. Ruchi khajuria, K. C.Gosswami, K.Singh,V. K. Dubey. Pattern of Lymphadenopathy on Fine Needle Aspiration Cytology in Jammu. 2006;8: (3):157- 59.

7. Gupta AK, Nayar M, Chandra M. Critical appraisal of fine needle aspiration Cytology in tuberculosis. Acta Cytol 1992 Jun; 36(3): 391-94.
8. Prasad RR, Narasimhan R, Sankran V, Veliath AJ. Fine needle aspration cytology in the diagnosis of superficial lymphadenopathy: an analysis of 2418 cases. Diagno Cytol 1996 Dec;15(5):382-86.

9. Paul PC, Goswami BK, Chakrabarty S, Giri A, Pramanik R. Fine needle aspiration cytology of lymph nodes - An institutional study of 1448 cases over a five year period J Cytology 2004;21:187-90.

10. Kline TS, Khannan V, Line IK. Lymphadenopathy and aspiration biopsy cytology review of 376 superficial lymph nodes. Cancer 1984; 54(6):1076-81.

11. Betsill WL, Hajdu SI. Percutaneous aspiration biopsy of lymph nodes. Am J. Clin Pathol 1980 April; 73(4): 471 - 79.

12. Shilpa G, Nataraju G. Pattern of Lymph Node Diseases in a Tertiary Level Referral Center: a cytological study of 943 cases. Int J Biol Med Res. 2013; 4(3): $3448-52$.

\section{How to cite this article?}

Kochhar A.K, Puri P.L, Kochhar S. Role of fine needle aspiration cytology in assessment of cervical lymphadenopathy. Int J Med Res Rev 2016;4(6):876-880doi: 10.17511/ijmrr.2016.i06.03. 